Archives de sciences sociales des religions

172 | octobre-décembre

Bulletin Bibliographique

\title{
La Fable mystique I et II : des lectures discontinues
}

\section{Patrick Goujon}

\section{OpenEdition}

Journals

Édition électronique

URL : http://journals.openedition.org/assr/27222

DOI : $10.4000 /$ assr.27222

ISSN : $1777-5825$

Éditeur

Éditions de l'EHESS

Édition imprimée

Date de publication : 1 octobre 2015

Pagination : 81-87

ISBN : 978-2-7132-2515-4

ISSN : 0335-5985

Référence électronique

Patrick Goujon, "La Fable mystique I et II : des lectures discontinues », Archives de sciences sociales des religions [En ligne], 172 | octobre-décembre, mis en ligne le 26 avril 2018, consulté le 03 mai 2019 URL : http://journals.openedition.org/assr/27222 ; DOI : 10.4000/assr.27222 


\section{Patrick Goujon}

\section{La Fable mystique I et II : des lectures discontinues}

La Fable mystique II, XVI ${ }^{e}$-XVII ${ }^{e}$ siècle, donne à vivre un moment étonnant de lecture. Venant à la suite du premier volume, l'écriture des textes du second pourtant précède ceux du premier parfois d'une large décennie. Il n'empêche, nous lisons ces textes après une trentaine d'années ${ }^{1}$. Notre lecture de Certeau aujourd'hui n'est plus celle des années 1980, et s'enrichit encore de La Fable mystique II. Il s'est noué entre Certeau et ses lecteurs un dialogue que relance ce livre dont la nouveauté vient en quelque sorte déplacer la réception de la réflexion de Certeau, en particulier sur la mystique, comme nous allons essayer de le montrer. Mais il s'agira bien ici du dialogue qui s'est noué avec Certeau et non pas d'un exposé de ce qu'apporterait, solitaire, cet opus posthumum. Entrer en dialogue nous semble d'autant plus pertinent qu'il s'agit d'une des interrogations majeures de Certeau : qu'est-ce qu'autorise un écrit mystique et quel conversar un écrit inaugure-t-il ? La Fable mystique II autorise cette conversation d'autant plus que plusieurs chapitres reviennent sur le travail de réception de la mystique par des mystiques, en particulier le chapitre 4, "Usages de la tradition ", mais aussi le troisième, "Le dire en éclats", et le septième, "La science expérimentale de la folie ». Mais le dialogue se noue entre ces chapitres non seulement du fait de leur étalement chronologique (de 1970 à 1985), non seulement parce qu'il y est question de la conversation mystique entre Surin et Jean de la Croix, mais aussi par l'usage de Jean de la Croix et de Surin que fait Certeau.

Nous procèderons ici en deux temps, en premier pour rappeler brièvement le cadre de La Fable mystique I, en tant qu'il donne à lire ce qui ne cesse de sortir de ce cadre. Dans un deuxième temps, nous engagerons un dialogue avec le deuxième volume qui interroge, encore une fois, le lieu de l'autre, en faisant venir au jour la discontinuité entre Jean de la Croix et Surin dans les analyses de Certeau, discontinuité qui ouvrira, je l'espère, un espace à notre réflexion.

1. Dans le champ de la mystique, dans les études de langue française, signalons Sophie Houdard, Les invasions mystiques : spiritualités, hétérodoxies et censures au début de l'époque moderne, Les Belles Lettres, 2008 ; François Trémolières, Fénelon et le sublime. Littérature, anthropologie, spiritualité, Honoré Champion, 2009. 
Certeau dessine un cadre pour observer la mystique. Il précise d'emblée qu'il sera débordé de tout côté ${ }^{2}$. Cet " excès " nous conduit au cœur de sa définition de la mystique. Ce cadre ( "la quadrature de la mystique ») est défini par quatre approches dont l'entrecroisement a fait la marque de la démarche certalienne.

En voici les quatre composantes : une nouvelle érotique, depuis que s'est emparée de l'Occident la nostalgie d'un Dieu unique objet d'amour ; une théorie psychanalytique : les questions posées par la mystique (le sujet, la pathologie des corps et des sociétés, le désir et l'amour) sont reprises et articulées dans cette autre discipline qu'est la psychanalyse. Un phénomène de réception au sens où Certeau va le définir. Troisième côté du cadre : l'historiographie. C'est là qu'apparaît la fondamentale "structure en miroir " de l'historiographie certalienne selon une symétrie " en trompe-l'œil », entre le mystique en quête du Dieu qui échappe et l'historien en quête du passé qui n'est plus ${ }^{3}$. L'interrogation épistémologique de Certeau ouvre à un mode d'exister ou en recouvre l'inquiétude. Quatrième côté : la fable, comme ces éclats des voix qui percent sous la chape de l'écrit qui recouvre l'âge moderne et dont les mystiques gardent la nostalgie.

Ce cadre permet à Certeau de "voir de quelle manière le sujet traverse la scène, lui échappe et coule ailleurs ". L'introduction à La Fable mystique I dessine avec force ce projet : les discours mystiques sont le langage d'une nostalgie, tant pour ceux qui les écrivaient hier que pour ceux qui les lisent aujourd'hui, mais pour d'autres raisons. Cette dimension nostalgique me paraît plus absente dans La Fable mystique II. Récits de la perte de l'Unique, les discours mystiques désignent l'étrangeté de notre propre place et "un désir de partir au pays ». L' " ouverture à une poétique du corps ", placée au terme de l'ouvrage, livre en résumé cette conclusion en forme de sentence : " est mystique celui ou celle qui ne peut s'arrêter de marcher et qui, avec la certitude de ce qui lui manque, sait de chaque lieu et de chaque objet que ce n'est pas ça, qu'on ne peut résider ici ni se contenter de cela. Le désir crée un excès. Il excède, passe et perd les lieux. Il fait aller plus loin, ailleurs. Il n'habite nulle part. Il est habité, dit encore Hadewijch, par "un noble je ne sais quoi ni ceci ni cela, qui nous conduit, nous introduit et nous absorbe en notre Origine" " ${ }^{4}$.

La Fable mystique I propose un cadrage impossible: le sujet toujours en échappée, son émergence à même son effraction. L'écriture en trace le voyage, jusque dans l'inscription des corps. On se souvient de l'épilogue où Certeau rapprochait le poème "Très Haut Amour » de Catherine Pozzi et le chant des chamans indiens.

2. Michel de Certeau, La Fable mystique I, XVI ${ }^{e}$-XVII ${ }^{e}$ siècle, [1982], Gallimard, coll. « Tel ", 1987, p. 12 (dorénavant, abrégé $F M$ I).

3. "Trompe l'œil » car, précise aussitôt Certeau, l'historien travaille à fournir des raisons là où le mystique fonde son existence sur ce qui lui échappe, $F M$ I, p. 21.

4. FM I, p. 408. 
«De même les chamans indiens partent dans la forêt aux bruits innombrables, en quête d'une musique - un chant d'oiseau ou de vent - qui fasse naître en eux ce qu'ils ne savent pas encore. On dit qu'un "esprit" les appelle. À cette "vocation", étrangement familière, ils passeront leur vie à répondre, une fois rentrés au village. Ainsi le poème "venait" à Catherine Pozzi, la nuit, sous sa forme presque définitive. La musique attendue et entendue résonne dans le corps à la manière d'une voix intérieure qu'on ne peut nommer et qui réorganise pourtant l'usage des mots. Qui en est "saisi” ou "possédé" se met à parler un langage hanté : la musique venue d'on ne sait où inaugure une autre rythmique de l'exister - certains disent : un nouveau "respir", une nouvelle façon de marcher, un autre "style" de vie. À la fois, elle capte une attention du dedans, elle trouble l'ordre des pensées, et elle ouvre ou libère de nouveaux espaces. Sans elle pas de mystique. L'expérience mystique a donc souvent l'allure d'un poème, qu'on "entend", comme on entre dans une danse. Le corps est "informé" (il reçoit la forme) de ce qui lui arrive ainsi bien avant que l'intelligence en ait la connaissance ${ }^{5}$. "

Ces lignes concentrent l'essentiel des conceptions certaliennes de la mystique en tant qu'elle en appelle à la voix. Après la lecture de La Fable mystique II, le dialogue entre ce chant et la poétique de Pozzi, apparaît comme typique du dialogue de réception, du mode de conversar dont Certeau traite à propos de Jean de la Croix et de Surin. La Fable mystique II permet de lire ce rapport aux voix de l'autre.

Dans le chapitre 4, intitulé « Usages de la tradition », publié en 1970, Certeau étudie le rapport de Surin à Jean de la Croix et place au cœur l'élaboration du concept de "la mystique " comme « énonciation ", le "modus loquendi ", qu'on retrouve dans La Fable mystique I. Certeau saisit ce rapport de Surin à Jean de la Croix en définissant la lecture comme "invention d'un sens ». "Toute lecture invente un sens » et est « pourtant docile au texte reçu mais sur le mode de dire ou la même chose en d'autres termes ou la même chose dans les mêmes termes " ${ }^{6}$. Certeau poursuit : la lecture " produit donc un nouvel état du texte " qui est le texte reçu. Certeau se donne alors ainsi d'étudier le Jean de la Croix de Surin. L'énonciation mystique articule ce qui d'une part donne lieu à un nouveau vocabulaire - chez Surin, le quasi-abandon du vocabulaire cosmique de Jean de la Croix, pour le langage technique, du brodeur, de l'horloge, une entrée du monde de la technique, des villes et de l'artisanat - et d'autre part l'invention de nouveaux sens des mêmes énoncés.

À partir de cette dynamique de la réception, Certeau esquisse un élargissement du problème. Selon Certeau, toute expérience spirituelle, dès lors qu'elle s'exprime, se trouve aliénée dans le langage. "L'auteur ou le sujet n'apparaît que soumis déjà à la loi d'une communauté " ${ }^{7}$. D'abord parce qu'elle emploie les mots des autres, et que, poursuit Certeau, "elle est soumise à un passage obligé, le tri et la pression du groupe ». L'homme ne parle qu'un langage reçu

\footnotetext{
5. Ibidem.

6. FM II, p. 166.

7. FM II, p. 167.
} 
d'autrui. Certeau conclut : " il n’y a pas à proprement parler d'expérience individuelle ${ }^{8}$. Les pages consacrées à Thérèse d'Avila dans la Faiblesse de croire avaient montré la fécondité de ce rapport entre langage, culture et expérience spirituelle, mais les analyses de La Fable mystique II, en mettant à jour une conception de l'individu toujours altéré par le langage, font entendre le regret, la nostalgie d'un individu qui tiendrait par lui-même. Illusion du sujet solipsiste ? Cet individu-là n'existe pas. Sans doute est-ce le deuil de la modernité, ou celui de Certeau.

Cette aliénation dans le langage est-elle la négation de l'expression de la nouveauté de l'expérience spirituelle, de sa singularité ? Comme le souligne Certeau, dans la lecture définie comme reprise par un sujet des mots d'un autre, il y a nouveauté parce que le texte est recouvert d'une autorité. Jean de la Croix intervient comme une autorité au sens où les textes de Jean de la Croix rendent possible un voyage. Certeau rapproche ces écrits mystiques de la littérature de voyage : dans la configuration épistémologique de la modernité, le fait raconté ouvre à de nouvelles expériences. Ce que les voyageurs ont vu ouvre de nouvelles possibilités ${ }^{9}$. Certeau lit ainsi la Science expérimentale de Surin : un fait raconté comme un savoir ou comme un croire possible rend possible un mode d'exister. Pour Surin, Jean de la Croix rend possible l'expérience, les «termes extraordinaires ", « l'objet verbal ", c'est-à-dire la possibilité de prendre position, d'user d'un langage, qui soit à la fois nécessaire et impossible ${ }^{10}$. Certeau reformule les dissimilitudes dissemblables, les oxymores de la langue mystique, cette "paix impétueuse » de Surin sur laquelle Certeau insiste tant. Les similitudes dissemblables fournissent à Certeau la matrice non seulement de l'analyse linguistique des phrases mystiques mais aussi du rapport du mystique à autrui et du rapport à l'existence. Des analyses de ces dissimilitudes, Certeau conclut que "le sens jaillit de l'impossibilité de s'arrêter à l'un ou l'autre des termes ». "Deux manques contraires définissent le mot mystique » : lorsque je dis la paix, manque la force impétueuse avec laquelle elle fond sur le sujet, mais en disant l'impétuosité manque l'effet de cette impétuosité qui n'est plus la tempête mais la paix. Le mot mystique se définit par les deux manques contraires ${ }^{11}$. L'énonciation mystique articule le manque des deux. L'énonciation fait entendre l'appel de l'autre. J'en appelle à l'autre pour signifier d'abord dans la langue.

Certeau creuse cet aspect dans l'étude des Dits mystiques de Jean de la Croix dans le chapitre 3 , intitulé «Le dire en éclats », qui fut d'abord une préface à

\section{Ibidem.}

9. Idem, p. 175. Voir sur ce rapprochement, Frank Lestringant, Le Huguenot et le sauvage, La controverse coloniale en France au temps des guerres de religion, Klincksieck, 1990; S. Requemora, Voguer vers la modernité. Le voyage à travers les genres au XVII siècle, Presses de l'université Paris-Sorbonne, 2012.

10. Idem., p. 186. Dorénavant, les références sont à la FM II.

11. p. 188. 
l'édition des Dichos donnée par Bernard Sesé en 1985. "En définissant les Dichos comme un lieu linguistique où advient une impétuosité ", Certeau revient sur la nature dialoguale de l'énonciation mystique. " La parole circule entre plusieurs, elle est interlocution au titre de ce qui manque à chacun. Elle est un plus pour tous en raison de ce que ces divers témoins ont en moins les uns par rapport aux autres ${ }^{12}$ ". Située non dans une analyse des énoncés mais des situations d'énonciation, la conversation est comprise comme la mise en relations de manquants. La conversation se nourrit de l'éclatement de la parole, de son impossibilité à tout saisir. "La diversification et dissémination de son énoncé et de sa force instaurent-elles l'échange collectif grâce auquel chacun reçoit de la parole ce qui lui fait encore défaut ${ }^{13}$ ". En même temps, Certeau peut affirmer qu'il n'existe aucun lieu particulier de la parole. On ne peut pas dire " elle est ici ", elle est en circulation du fait de ceux qui ceux qui parlent sont des sujets manquants. En poursuivant l'analyse des Dichos, Certeau conclut que la parole crée un lieu, «le lieu même de cette interlocution. À la façon d'une place publique ou d'un lieu commun, il crée un espace de liberté. Il parle, c'est-à-dire qu'il y a de l'autre en lui ${ }^{14}$ ». Cette présence de l'autre, son élocution à même le sujet, Certeau n'a cessé de le penser, de le traquer avec Surin et en particulier dans le voyage de la folie que raconte la Science expérimentale à laquelle il va consacrer ce chapitre 7, publié en 1984, "La science expérimentale de la folie ».

Ici, l'éblouissement pour Surin n'est plus seulement en raison de la manière dont Surin est figure de la mystique mais en raison de ce qu'il est figure de la folie à laquelle s'articule de manière cachée, refoulée, la raison de la conscience moderne occidentale. Cette historicisation de Surin permet le dialogue avec la conscience moderne occidentale dans cette scission de la raison et de la folie.

Analysé comme un récit de voyage, la Science Expérimentale égraine les déplacements du narrateur entre deux pôles, ce qui est le propre de tout récit de voyage. Le récit de voyage est structuré entre un ici et un là-bas, l'ici du départ, et là-bas rêvé, que l'on atteint par étapes ou que l'on n'atteint pas, qui est autant l'aventure maritime que l'aventure missionnaire ${ }^{15}$. Dans la Science Expérimentale, cette double polarité est constituée par la scission du je, entre un « je » et un « il », « index littéraire de la folie », le « je », sujet écrivant, et le « il », l'objet raconté, le fou et le damné, sans confusion. Le récit gère alors la relation entre ces deux espaces hétérogènes et le récit est l'histoire du progrès du « je » dans la région aliéné du «il », car le je va vers le «il ». Cette histoire s’écrit comme l'histoire de l'écriture qui permet le retour à moi de cet il autre. C'est dans l'écriture que je va vers le il, opération thérapeutique, écrit M. de Certeau, et il

\author{
12. p. 153 . \\ 13. Ibidem. \\ 14. p. 157. \\ 15. Bernard Forthomme, Théologie de l'aventure, Le Cerf, 2013.
}


précise, "et/ou psychanalytique ${ }^{16}$ ". Certeau corrige aussitôt, écriture qui est " en réalité reconquista des énoncés par le locuteur ", note intéressante si l'on songe à ce que serait l'écrit dans la cure psychanalytique, et à ce que fut l'écrit - et son statut discuté - dans la direction des âmes inspirée des Exercices spirituels d'Ignace de Loyola.

À ce point, Certeau pense la genèse du sujet croyant en tant que pouvoir croire. J'en reprends le cheminement. Le sujet naît de pouvoir croire. Dans la Science Expérimentale, comme dans tout récit de voyage, il est question des choses étranges si peu croyables. Point de départ de la réflexion. Que ferait un auteur de récit missionnaire? Il s'efforcerait de faire croire ce qu'il nous donne à lire en le rendant vraisemblable en faisant en sorte que l'extraordinaire ne soit plus qu'une merveille, ce qui a un effet de grossissement, ou d'amincissement. La problématique de la Science Expérimentale est autre, dit Certeau: elle concerne non la vraisemblance mais plutôt la possibilité ou l'impossibilité de croire. La Science Expérimentale répond à la question : est-il possible ou non de croire $^{17}$ ?

On retrouve en quelques pages un précipité des modalités du croire, du pouvoir, du savoir et du vouloir tel qu'elles sont exposées dans La Fable mystique I à propos de Thérèse d'Avila et de Maître Eckhart. "Le vouloir est le ressort permanent du parcours de Surin. Surin ne veut qu'une seule chose : se conformer à la volonté de Dieu. Il veut le vouloir de l'autre. Mais comment accéder à ce vouloir de Dieu ? » Certeau pense que l'énoncé de base serait « je veux faire ce que je crois que Dieu veut et entre les deux vouloirs, le croire fait barre ». La question est bien de décider s'il est croyable de croire, ou comme l'écrit Certeau, "possible de pouvoir ». C'est dans le geste physique d'écrire que Certeau situe cette ouverture de possible pouvoir, du croire croyable où l'écriture est le lieu par lequel Surin accède à un "je veux » qui ne le scinde plus entre un "je » et un « il ». Or, curieusement, au sens où moi je trouve cela curieux, Certeau, en commentant ce passage de la Science Expérimentale, ne revient pas sur ce qui me semble fondamental chez lui quand il analyse le Jean de la Croix.

En effet, le passage à l'écriture de Surin a lieu dans le récit d'une conversation avec le confesseur, or, partout, cette conversation chez Certeau disparaît. Il me semble que cette disparition éclaire la discontinuité de la lecture de Surin et de Jean de la Croix par Certeau. Si Certeau analyse l'expérience d'écriture comme thérapie, et comme avènement du sujet dans un espace nouveau, cet espace a été autorisé par une parole, au sens que Certeau donne à cette autorisation dans l'analyse des Dichos. Non pas la parole d'un seul, du confesseur, encore moins la parole de Dieu, mais dans une interlocution, dans l'échange avec le confesseur

16. p. 242.

17. p. $242-245$. 
qui fait naître en Surin une parole intérieure qui est entendue ensuite comme si elle venait de Dieu. L'espace entre l'énonciation et la réception est la condition de cette genèse du sujet comme sujet croyant. Ce qui fait d'un sujet un sujet croyant tient dans l'écriture de ce qu'a été une scène d'interlocution, et pas simplement dans l'écriture, l'interlocution ayant autorisé de pouvoir croire. Or ce schéma est ce que décrit précisément Certeau à propos de Jean de la Croix ; Surin donne à le lire dans la Correspondance, éditée par Certeau, mais il disparaît de la plume de Certeau quand il analyse Surin. Dans la Correspondance, il y a constamment ouvert un espace entre l'énonciation et la réception. Dans cet espace se trouve la condition de la conversation et de la correspondance dont la puissance va jusqu'à rendre possible la réception d'un texte comme parole ${ }^{18}$. Le texte lu peut se faire interpellation : il s'adresse de l'extérieur de moi à ce qui en moi est au plus intime de moi-même et se révèle seulement à cet appel. Puissance, virtù, comme écrirait Certeau, qui n'est autre que celle de la voix qui m'est adressée par autrui et qui m'appelle, voix paradoxale dans la lecture. Cette voix n'est pas la voix des chamans indiens qu'on va quêter dans la forêt, mais la voix que l'on quête dans l'écrit, et qui peut s'entendre pour le sujet qui croit qu'il peut croire. Ce que je lis m'est offert avec la force ou la douceur d'un autre que je découvre en moi et qui m'appelle, instaurant la présence d'autrui dans le dialogue et non la fusion. La littérature épistolaire, comme l'avait écrit Certeau, est le lieu par excellence de cet entrecroisement dont il nous donne à lire la force dans le passage du voir au goûter ${ }^{19}$.

La lecture et l'expérience mystique pour Certeau, telles que Surin lui permet de les penser, ne sont plus du côté du voir, mais du côté du goût, le sens du proche qui contredit la vue, le sens du lointain, et conjugue, dit Certeau, une érotique à une connaissance. "Cette expérience de muqueuse est de type épistémologique (elle concerne une "certitude”), mais une épistémologie fondée sur un toucher et articulée sur un discernement des plaisirs. Elle suppose au tact, c'està-dire à une fonction érotique indissociable d'une relation à l'autre, une valeur de connaissance que n'a pas une relation visuelle à un objet ».

Si ce que Certeau écrit là me convainc, je pousse alors une conclusion qu'il n'a pas écrite : ce sens de l'autre qui est donné là n'est pas celui d'une absence mais celui d'une présence.

Patrick GOUJON

Centre Sèvres

patrick.goujon@jesuites.com

18. Je me permets de renvoyer ici à mon étude Prendre part à l'intransmissible. La communication spirituelle à travers la correspondance de J.-J. Surin, Jérôme Millon, 2008.

19. FM II, p. 250, de même pour la référence suivante. 
